\title{
Design and Fabrication of Coplanar YBCO Structures on Lithium Niobate Substrates
}

\author{
E. Rozan, C. Collado, A. Garcia, J.M. O'Callaghan, R. Pous \\ Universitat Politècnica de Catalunya, Campus Nord UPC D3, Barcelona 08034, Spain \\ L. Fabrega, J. Rius, R. Rubí, J. Fontcuberta \\ Institut de Ciència de Materials de Barcelona-CSIC, Campus UAB, Bellaterra 08193, Spain \\ F. Harackiewicz \\ Southern Illinois University, Dpt of Electrical Engineering, Carbondale 62901, Il
}

\begin{abstract}
YBa}_{2} \mathrm{Cu}_{3} \mathrm{O}_{7-\delta}(\mathrm{YBCO})$ with low $\mathrm{RF}$ losses has been successfully deposited onto lithium niobate (LNO) to improve the performance of electrooptic Mach-Zender modulators. Epitaxial, c-axis oriented superconducting YBCO thin films have been grown on X-cut LNO single crystals with a yttriastabilized zirconia (YSZ) buffer layer by RF magnetron sputtering. This buffer layer is needed to obtain good superconducting properties of the YBCO grown.

Numerical tools have been developed to analyze CPW structures based on YBCO/YSZ/LNO trilayers, and they indicate that YSZ thickness has to be kept to the minimum necessary for good YBCO growth. With this restriction, the RF losses of YBCO/YSZ/LNO samples have been measured. The results from these measurements are used to quantify the performance enhancement in a Mach-Zender modulator using YBCO electrodes.
\end{abstract}

\section{INTRODUCTION}

The use of high temperature superconducting (HTS) films deposited onto lithium niobate (LNO) single crystals might lead to enhanced performance in a number of acoustic and photonic devices. One of them is the electrooptical (EO) Mach-Zender external modulator, for which predictions of improved performance already exist when its metal electrodes are substituted by superconductive ones [1]. These electrodes are arranged in a coplanar waveguide (CPW) structure, which has to meet stringent requirements in both its effective propagation index and characteristic impedance.

In spite of the predictions in [1], no measured data is easily found in the literature on a Mach-Zender device made with HTS films, not even on simple RF measurements made on HTS lines on LNO substrates. To cover these issues, this work addresses the problem of fabricating $\mathrm{YBa}_{2} \mathrm{Cu}_{3} \mathrm{O}_{7-\delta}$ (YBCO) $\mathrm{CPW}$ lines on LNO substrates with low losses and predictable RF properties (effective propagation index and characteristic impedance), and updates the performance predictions of the Mach-Zender external modulator based on the measured RF performance of the CPW lines made.

Manuscript received September 15, 1998.

This work is supported by Red Eléctrica de España (REE), and Cables Pirelli S.A.

\section{MATERIAL DEPOSITION}

Superconducting YBCO thin films have been grown on Xcut LNO single crystals using YSZ as buffer layer. This buffer is needed due to the poor structural and chemical compatibility of LNO and YBCO. Its use should improve the growth quality and thus the film microstructure, resulting in low surface resistance and high critical currents necessary in most applications. For Mach-Zender modulators, the buffer layer has to allow the epitaxial growth of YBCO, and has to have moderate permittivity at optical wavelengths. YSZ has been chosen as buffer layer since it meets both requirements.

The YSZ and YBCO films were deposited by on-axis RF magnetron sputtering, using home-made, stoichiometric targets; the YSZ one contained $16 \% \quad \mathrm{Y}_{2} \mathrm{O}_{3}$. Optimal deposition conditions for YSZ are 50mTorr of total pressure, with $38 \% \mathrm{O}_{2}$ and $62 \% \mathrm{Ar}$, substrate temperature $700-750^{\circ} \mathrm{C}$ and RF power of $50 \mathrm{~W}$. For superconducting $\mathrm{YBCO}$, the growth conditions had to be changed to higher temperatures, $780^{\circ} \mathrm{C}$, and higher total pressure, $300-400 \mathrm{mT}$ orr; the $\mathrm{O}_{2}: \mathrm{Ar}$ pressure ratio was kept almost constant. The samples were quenched to $500^{\circ} \mathrm{C}$ in the growth atmosphere, and then slowly brought to room temperature under 500Torr of $\mathrm{O}_{2}$.

Figure 1 shows the $\theta-2 \theta$ X-ray diffraction (XRD) patterns of a YSZ/LNO film and a YBCO/YSZ/LNO heterostructure grown on a substrate at $700-750^{\circ} \mathrm{C}$ and with $50 \mathrm{~W}$ of RF power. The YSZ layer grows along the (100) direction; the aaxis spread perpendicular to the film, estimated from the full

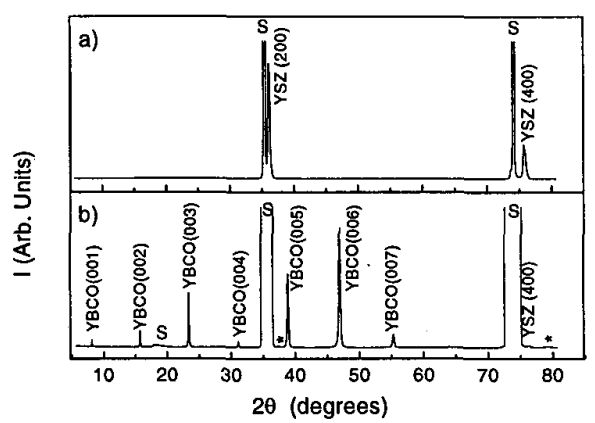

Fig. 1. $\theta-2 \theta X$-ray diffractograms of a YSZ/LNO film (a), and a YBCO/YSZ/LNO heterostructure (b). Unknown peaks are denoted by a *. Substrate peaks are labeled with a $S$ 
width at half maximum of the $\omega$-scan for the (400) peak, is of only $0.3^{\circ}$. The optimal thickness of the buffer layer turned out to be around $80 \mathrm{~nm}$. On the top of the (100) YSZ layer, the YBCO film grows along the c-axis direction, i.e. with the superconducting $\mathrm{Cu}-\mathrm{O}$ planes parallel to the film surface, as intended; $\omega$-scans for the (006) peak indicate a c-axis spread of the order of $1^{\circ}$, for $100 \mathrm{~nm}$-thick films. As it can be appreciated in Fig. 1, the XRD of the YBCO/YSZ/LNO heterostructure reveals that, after the YBCO growth, the YSZ layer suffers a significant crystalline deterioration, and crystals with (111) orientation are observed. This translates in a noticeable granularity in the YBCO film, which has been observed by AFM.

The superconducting critical temperature $T_{c}$ of the YBCO films was measured by inductive and transport methods (Fig.2). The best films display a $\mathrm{T}_{\mathrm{c}} \sim 88 \mathrm{~K}$, obtained from the resistance drop, and transition widths of $3-4 \mathrm{~K}$.

\section{NUMERICAL SIMULATION}

Numerical simulations have been employed to determine the microwave parameters (characteristic impedance, effective propagation index, attenuation constant) of the CPW line (Fig. 3). Several features of the structure in Fig. 3 introduce difficulties in these simulations: the presence of two dielectric materials (YSZ and LNO), the strong anisotropy of one of them (LNO), the high permittivities involved, and the complex conductivity of the HTS film. A two-step approach is used. As a first approximation, quasistatic simulations have been done, which do not take into account the features of the HTS material, and do not yield data on the dispersive behavior of the structure (which is expected to be negligible, since the dimensions of the structure are small compared to the wavelength). These quasi-static results are later confirmed by a full-wave analysis.

The quasi-static formulation used the Point Matching Method (PMM) - is based on [2], with the addition of a topplate. The algorithm yields the capacitances per unit of length for the structure in Fig. $3(C)$ and for the same electrode

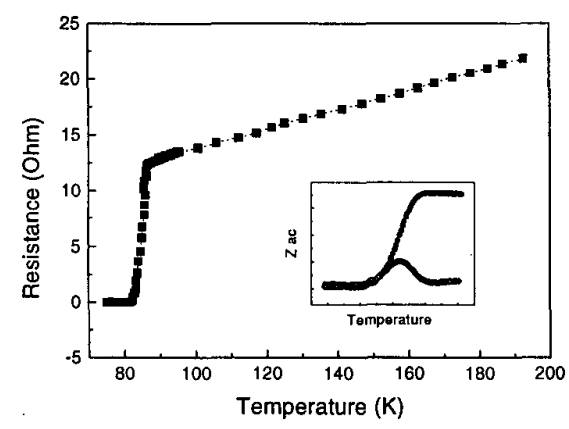

Fig. 2. Temperature dependence of resistivity of a superconducting YBCO/YSZ/LNO heterostructure. Inset: Superconducting transition measured by a mutual inductance technique.

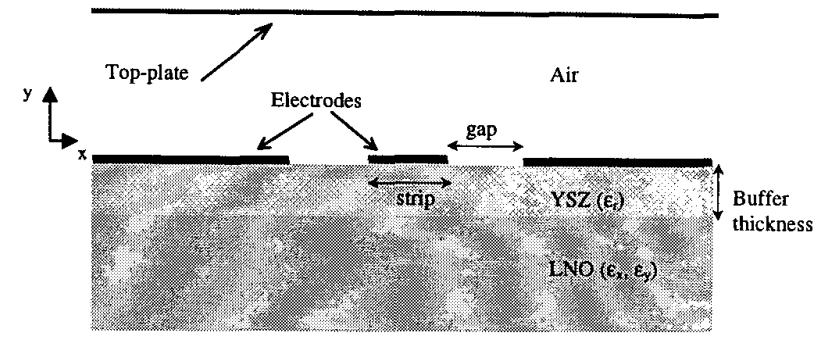

Fig. 3. CPW configuration. YSZ dielectric constant is 29, LNO dielectric constants are $\varepsilon_{\mathrm{x}}=\varepsilon_{\mathrm{z}}=43$ and $\varepsilon_{\mathrm{y}}=28$.

configuration embedded in vacuum $\left(C_{o}\right)$. From them, the effective propagation index $\left(n_{m}\right)$ and the characteristic impedance $\left(Z_{0}\right)$ are calculated:

$$
\begin{gathered}
n_{m}=\sqrt{\frac{C}{C_{o}}} \\
Z_{0}=\frac{1}{c_{o} \sqrt{C C_{o}}}
\end{gathered}
$$

where $c_{o}$ is the speed of the light in the vacuum. For a metallic electrode, the attenuation can be calculated using its conductivity and the values of $n_{m}$ and $Z_{0}$ in an analytical formula [3, p 419].

To confirm the low dispersion behavior of CPW electrodes at microwave frequencies, the structure in Fig. 3 was analyzed using the spectral domain approach (SDA) [4]. This general method has been modified to account for the anisotropy in the substrate layer [5], and for the presence of a top-plate. To take advantage of the formulation, and to include the effect of the superconductor surface impedance, a complex boundary condition is introduced in the original Green's functions system:

$$
\left[\begin{array}{cc}
\tilde{Z}_{x x}-Z & \tilde{Z}_{x z} \\
\tilde{Z}_{z x} & \tilde{Z}_{z z}-Z
\end{array}\right]\left[\begin{array}{l}
\tilde{J}_{x} \\
\tilde{J}_{z}
\end{array}\right]=\left[\begin{array}{c}
\tilde{E}_{x} \\
\tilde{E}_{z}
\end{array}\right]
$$

Defining $\lambda$ as the two-fluid model penetration depth for superconductors, $Z$ in (3) becomes $1 / \sigma$ if $t<<\lambda$, otherwise $Z_{s} \operatorname{coth}(t / \lambda)$. In the case of a normal metal, the conductivity $\sigma$ is real and the skin depth $\delta$ is used instead of $\lambda$.

To compare both methods, and to confirm the low dispersion in the device, quasi-static and full-wave simulations have been done on a $100 \mu \mathrm{m}$ gap and $50 \mu \mathrm{m}$ strip CPW structure with gold electrodes. Furthermore, to obtain an experimental confirmation, such a structure was fabricated and measured on a Cascade Microtech Summit-9000 waferprobing station. Both measurements and simulations yielded a constant value of $n_{m}$ and $Z_{0}$ from 5 to $20 \mathrm{GHz}$. Results are summarized in Table I.

Further simulation showed that changes in $Z_{0}$ and $n_{m}$ are negligible if the gold electrodes are replaced by a HTS superconducting layer. 
TABLE I

$\mathrm{Z}_{0}$ AND $n_{m}$ FOR $100 \mu \mathrm{m}$ GAP $50 \mu \mathrm{m}$ STRIP CPW

\begin{tabular}{cccc}
\hline & Quasi-static & $\begin{array}{c}\text { Full-Wave } \\
(20 \mathrm{GHz})\end{array}$ & $\begin{array}{c}\text { Measurement } \\
(20 \mathrm{GHz})\end{array}$ \\
\hline$Z_{0}[\Omega]$ & 44.70 & 46.33 & 44.11 \\
$n_{m}$ & 4.091 & 4.234 & 4.103 \\
\hline
\end{tabular}

Once the simulations tools were developed, they were applied to the design of the CPW structure, and in particular to analyze the effects of the buffer thickness. Standard EO devices employ a $\mathrm{SiO}_{2}$ buffer $\left(\varepsilon_{\mathrm{T}}=3.8\right)$ to match the effective propagation index of the electrical and optical wave. In our case, the choice of a buffer material (YSZ, with $\varepsilon_{\mathrm{r}}=29$ ) is much more restricted, so -as our simulations showed- the ability to match these indexes is severely restricted (Fig. 4). Thus, the YSZ thickness has been kept to a minimum (80nm) to allow adequate growth of the YBCO layer. Also, alternative methods of velocity matching will have to be used if a YBCO/YSZ/LNO heterostructure is used in a $\mathrm{EO}$ modulator [6].

Since the operating bandwidth of an EO device is strongly limited by the CPW attenuation, simulations of line attenuation in both gold and YBCO CPW line have also been studied. Table II indicates that a significant reduction in conductor losses can be obtained by using superconductor. As detailed later, these losses are dominant in normal metal electrodes, thus device performance should improve with their reduction.

\section{RF PERFORMANCE}

As mentioned earlier, the RF losses are the main limiting factor in the performance of an EO modulator, provided that the velocities of the optical and microwave signals are matched. Therefore, to prove that high frequency losses are reduced using superconducting materials, CPW one-port, half-wave resonators have been built using both YBCO and gold electrodes. Resonant frequency has been chosen at about $7 \mathrm{GHz}$ to fit the resonators within $10 \times 10 \mathrm{~mm}$ substrates. The size of the CPW electrode was designed to obtain a $50 \Omega$ impedance $(200 \mu \mathrm{m}$ gap and $50 \mu \mathrm{m}$ strip). Attenuation constants are extracted from the measured quality factors $Q_{0}$ by using:

TABLE II.

GOLd AND YbCo ATTENUATION CONSTANT. (No DIELECTRIC OR RAdiATION LOSSES INCLUDED).

\begin{tabular}{cccc}
\hline & $300 \mathrm{~K}$ & $77 \mathrm{~K}$ & $55 \mathrm{~K}$ \\
\hline $\mathrm{Au}\left[\mathrm{Np} / \mathrm{m} \cdot \mathrm{GHz}^{-1 / 2}\right]$ & 1.19 & 0.59 & 0.49 \\
$\mathrm{YBCO}\left[\mathrm{Np} / \mathrm{m} \mathrm{GHz}^{-2}\right]$ & - & $1.5 .10^{-3}$ & $1.6 .10^{-4}$ \\
\hline
\end{tabular}

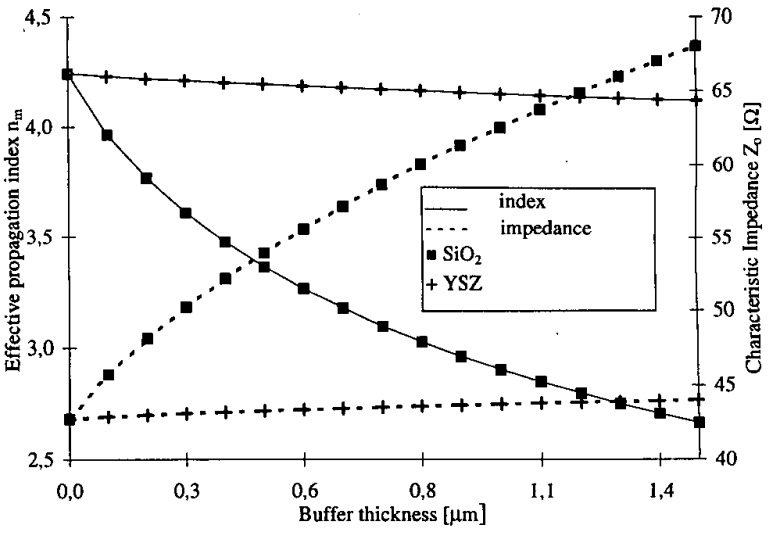

Fig. 4. Dependence of of $Z_{0}$ and $n_{m}$ on buffer thickness. Buffer materials are: $\mathrm{SiO}_{2}$ and $\mathrm{YSZ}$.

$$
\alpha_{i}=\frac{\pi}{2 l_{e f f} Q_{0}}
$$

where $l_{\text {eff }}$ is the effective length of the resonator.

Fig. 5 summarizes the experimental results:

- At $55 \mathrm{~K}$, the measured quality factor gain between a CPW YBCO resonator and an identical resonator made with gold is 2.8 times, indicating that the total attenuation is reduced by the same factor as observed in Fig. 5 . However, in the YBCO resonator, no significant loss improvement is observed below that temperature.

- At sufficiently low temperature (below 55K), losses due to superconductors are dominated by other types of losses (dielectric and radiation). This is in good agreement with the gold resonator data, in which the difference between the measured overall attenuation constant, and the one that can be predicted for gold, gives a value of residual losses close to the total losses in the superconducting resonator.

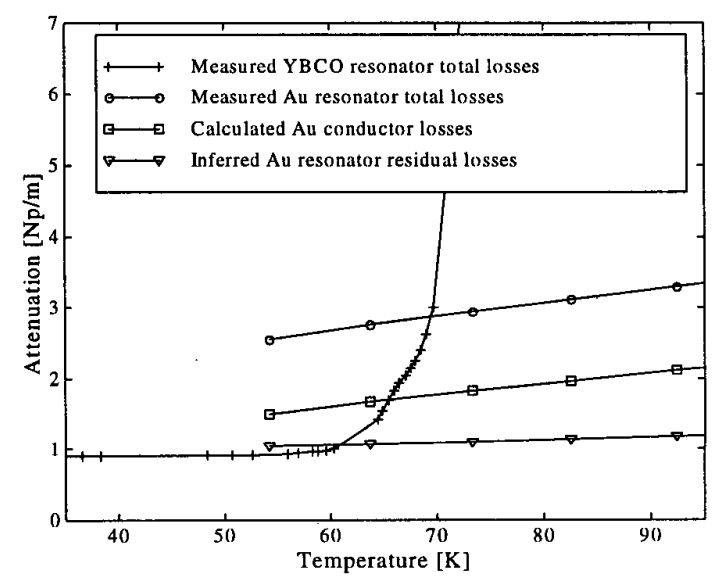

Fig. 5. Attenuation constants of gold and YBCO CPW resonators at $7 \mathrm{GHz}$. 
Similar comparative measurements made on CPW gold resonator with and without YSZ have confirmed that no significant RF losses were introduced by the buffer layer.

\section{EO DEVICE PERFoRMANCES PREDICTION}

From the RF measurements and the simulation tools developed, an updated analysis of EO Mach-Zender external modulator performance can be done.

The modulator bandwidth is defined from the modulation depth [7]

$$
F(f)=\sqrt{\frac{e^{-2 \alpha L}-2 e^{-\alpha L} \cos \theta+1}{(\alpha L)^{2}+\theta^{2}}}
$$

as the frequency $f$ for which $F(f) / F(0)=1 / \sqrt{2}$. $L$ represents the interaction length of the modulator and $\alpha$ the total attenuation line. $\theta$ is the difference between the optical and electrical field propagation speeds .

The figure of merit [1] is defined as the ratio between the bandwidth and the power necessary to obtain a $180^{\circ}$ phase shift, $P=V_{\pi}^{2} / 2 Z_{0}$, with $Z_{0}$ the line impedance and

$$
V_{\pi}=\frac{\lambda}{2 r_{33} n_{o}^{3} L \Gamma}
$$

the half-wave voltage. In (6), $\lambda$ is the free space optical wavelength, $r_{33}$ and $n_{o}$ are LNO optical parameters and $\Gamma$ the electrical-optical overlap parameter [8].

The predictions have been done using a modulator whose CPW electrode has a $50 \mu \mathrm{m}$ strip and a $10 \mu \mathrm{m}$ gap, considering no walk-off. The improvement due to the introduction of the superconductors is significant and the benefits may increase the bandwidth from $4 \mathrm{GHz}$ up to 40 $\mathrm{GHz}$ for a $5 \mathrm{~cm}$ interactive length. The figure of merit shows (Fig. 6) that the use of YBCO electrodes is advantageous with respect to gold ones for electrode lengths longer than 1.6 $\mathrm{cm}$, and the performance improvement extends as longer electrodes are fabricated.

\section{CONCLUSION}

YBCO with low RF losses has been successfully deposited onto an electrooptic substrate (LNO) to improve the performance of EO Mach-Zender modulators. To obtain good superconducting properties, a YSZ buffer is needed between the LNO and the YBCO. It is found that a substantial improvement in bandwidth can be obtained, provided that the velocity of the optical and electrical signals is matched. Unlike in the conventional technology, this velocity matching cannot be made by adjusting the buffer thickness, so other matching alternatives will have to be pursued.

\section{ACKNOWLEDGMENT}

The authors would like to express their appreciation to $\mathrm{Dr}$ Dima Petrov and Dr Lluís Torner for their encouraging discussions and helpful remarks. The technical assistance of Ing. Aldo Perasso (Pirelli Cavi) has been also of great help.

\section{REFERENCES}

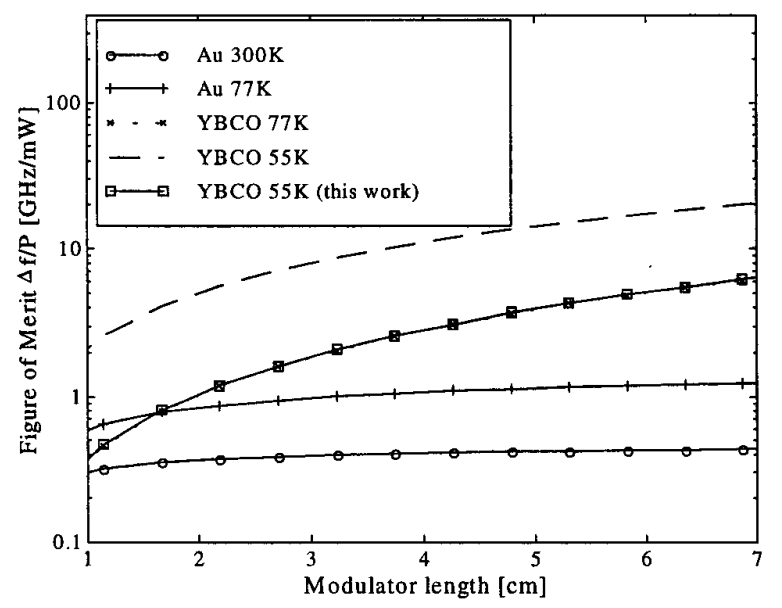

Fig. 6. Figure of merit of a walk-off compensated EO modulator, as a function of modulator length and with several types of electrode materials: Gold, state of the YBCO (on $\mathrm{SrTiO}_{3}$ substrate), YBCO obtained in this work. This figure of merit includes an extrapolation of the frequency dependence of the RF losses in each type of material. The trace of the state of the art YBCO at $77 \mathrm{~K}$ and of the YBCO produced in this work at $55 \mathrm{~K}$ are overlapped.
[1] K. Yoshida, K. Ikeda, K. Saito and Y. Kanda, "Application of Superconductivity Striplines to Traveling-Wave Type $\mathrm{LiNbO}_{3}$ Optical Modulator", IEEE Trans. On Applied Superconductivity, vol. 3, No. 1, pp. 2792-2795, Mar. 1993.

[2] D. Marcuse, "Electrostatic Field of Coplanar Lines Computed with the Point Match Method", IEEE Journal of Quantum Electronics, vol. 25, No. 5, pp. 939-947, May 1989.

[3] K.C. Gupta, Garg, Bahl "Microstrip Lines and Slotlines", Bhartia. Second Edition, 1996, Artech House.

[4] Tatsuo Itoh, Numerical Techniques for Microwave and MillimeterWave Passive Structures, Chapter 5, "Spectral Domain Approach," pp. 335-380, John Wiley \& Sons, New York, 1989.

[5] Z. Cai and J. Bornemann, "Generalized Spectral-Domain Analysis for Multilayered Complex Media and High- $\mathbf{T}_{\mathbf{c}}$ Superconductor Applications", IEEE Trans. on Microwave Theory Tech., vol. 40, No. 12, pp. 2251-2257, Dec. 1992.

[6] K. Kawano, K. Noguchi, T. Kitoh and H. Miyazawa, "A Finite Element Method (FEM) Analysis of a Shielded Velocity-Matched Ti:LiNbO3 Optical Modulator", IEEE Photonics Tech. Letters, vol. 3, No. 10, pp. 919-921, Oct. 1991. Travelling-Wave $\mathrm{LiNbO}_{3}$ Interferometric Modulators", IEEE Joumal of Quantum Electronics, vol. 27, No. 3, pp. 608-617, Mar. 1991.

[8] R. Alferness, "Waveguide Electrooptic Modulators," IEEE Trans. on Microwave Theory Tech., vol. 30, No. 8, pp. 1121-1136, Aug. 1992.
17] H. Chung, W. Chang and E. Adler , "Modeling and Optimization of 